\title{
CONTROLADOR DE TEMPO QUASE ÓTIMO PARA SISTEMAS BIDIMENSIONAIS
}

\author{
Leandro Pfuller lisboa*, Henrique Lasevich*, Nelso Bedin*, Jeferson Vieira Flores*, \\ Aurélio Tergolina Salton* \\ *PUCRS - Grupo de Automação e Controle de Sistemas \\ Av. Ipiranga, 6681, 90619-900 \\ Porto Alegre (RS), Brasil
}

Emails: \{leandro.lisboa, henrique.laevich, nelso.bedin\}@acad.pucrs.br, \{jeferson.flores, aurelio.salton\}@pucrs.br

\begin{abstract}
This paper focuses on the development of a proximate time optimal control method for two dimensions rigid body systems. Our approach is based on the traditional Proximate Time-Optimal Servomechanism (PTOS), which starts with a near-time-optimal controller and then switches to a linear controller at the time when the system output approaches a given target. This paper proposes to expand the PTOS, a unidirectional control law to a two dimensional systems, in order to perform linear point to point trajectories.
\end{abstract}

Keywords - Bidimensional Rigid Body System, Nonlinear Feedback, Time-Optimal Performance.

Resumo- Este artigo foca no desenvolvimento de um controlador de tempo semi ótimo para sistemas de corpo rígido bidimensionais. A abordagem baseia-se na tradicional técnica de controle Proximate Time-Optimal Servomechanism (PTOS), a qual inicia com uma aproximação do Time-Optimal Control (TOC) e, em seguida, comuta para um controlador linear Proporcional Derivativo (PD), no instante em que a saída do sistema se aproxima da referência. Este artigo propõe uma expansão do PTOS, uma lei de controle específica para sistemas unidimensionais, para ser utilizado em sistemas bidimensionais a fim de realizar trajetórias lineares ponto a ponto.

Palavras-chave - Sistema Bidimensional, Controlador Não Linear, Performance de Tempo Ótimo.

\section{Introdução}

Atuadores de posição trabalhando em malha fechada têm como objetivo alcançar a referência com a melhor precisão menor tempo possíveis. Como exemplo desta aplicação, existem atuadores atuando como Pick and Place, Hard Disk Drives (HDD), Braços Robóticos, Estações de Montagem Automática entre outras inúmeras aplicações de precisão na engenharia.

A maior parte dos atuadores usados na engenharia de precisão são modelados com equações de dinâmica de corpo rígido, tais como motores eletromagnéticos, (Grabner et al., 2010); braços robóticos, (Geering et al., 1986); sistemas HDD's de simples estágio, (Chen, 2006) bem como duplo estágio,(Zheng et al., 2010), entre outros.

Conforme (Zheng et al., 2010), desempenho em tempo ótimo, ou, tempo mínimo de movimentação de um ponto inicial a um ponto final é o principal objetivo de inúmeros sistemas de controle. Assim, a técnica que obtém o melhor desempenho em servomecanismo é a Time Optimal Controller (TOC). Este controlador normalmente é chamado de Bang-Bang Control devido a sua atuação ser baseada em uma máxima aceleração seguida de uma máxima desaceleração possível. Esta ação é consideravelmente agressiva, o que resulta em um fenômeno conhecido como Chatering, (Khalil, 2002), tornando o controlador TOC incapaz de fornecer uma solução em um sentido prático. Dado este cenário, diferentes técnicas de controle tem sido sugeridas na literatura para a obtenção de uma aproximação de tempo ótimo. A qual inclui Proximate Time-Optimal Servomechanism control (PTOS), (Workman et al., 1987b), (Pascoal et al., 1989), (Salton et al., 2012) e (Workman et al., 1987a); Variable Structure Sliding Mode control, (Hung et al., 1993); Nonlinear control, (Chen et al., 2003); Mode Switching control, (Yamaguchi et al., 1998); Two Degrees of Freedom control, (Yi and Tomizuka, 1999); Linear Quadratic Gaussian control(LQG), (Hanselmann and Engelke, 1988) e Adaptive control, (Serikitkankul et al., 2005).

Do ponto de vista prático, a técnica de controle mais usual é o PTOS, o qual fornece uma solução relativamente simples e elegante para uma aproximação de tempo ótimo em movimentação ponto a ponto. A técnica PTOS foi desenvolvida com o objetivo de resolver o fenômeno de chattering. Esta técnica consiste em uma troca suave da lei TOC no instante em que o sinal se aproxima da referência para uma lei de controle linear, evitando assim, uma mudança abrupta no sinal de controle. Como consequência, esta lei de controle utiliza a máxima aceleração do atuador apenas quando lhe é conveniente, tornando-a ideal para sistemas unidimensionais. Contudo, quando a intenção é atuar em sistemas bidimensionais esta técnica não garante a sincronia dos eixos, não sendo capaz de alcançar uma trajetória linear bidimensional ponto a ponto.

Este artigo apresenta o desenvolvimento de uma nova abordagem referente ao PTOS, tornando possível utilizá-lo em um sistema de corpo 


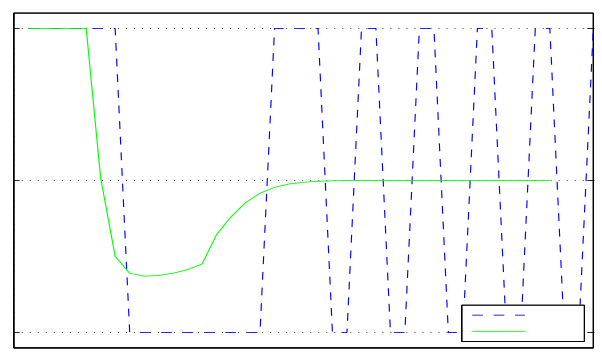


- Aplica-se uma lei de controle que comute da lei não linear complexa para uma lei linear simples, tal como um PD, quando o sistema se aproxima da referência, conseguindo assim uma estabilidade assintótica.

Estas três etapas resultam em um controlador dado por (3), onde o sinal de controle $u$ tem o comportamento apresentado na Figura 1.

$$
u(t)=k_{2}\left(-f_{P}(e)-\dot{y}\right)
$$

onde:

$f_{P}(e)= \begin{cases}\left(k_{1} / k_{2}\right) e & ,|e| \leq y_{l} \\ \operatorname{sgn}(e)(\sqrt{ } & \end{cases}$ 


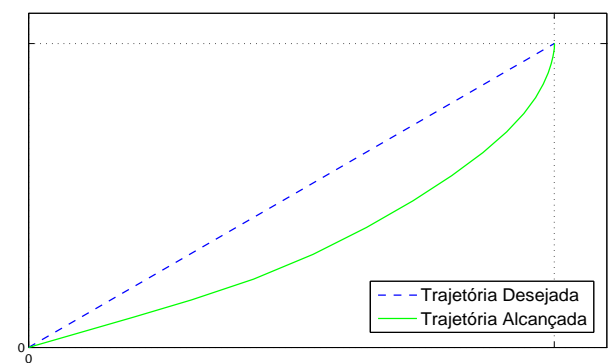




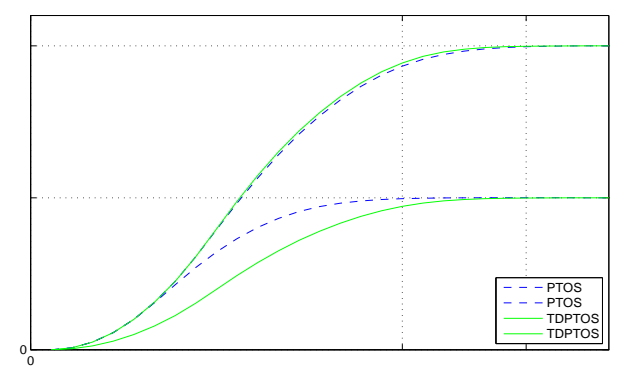


